\title{
Alternativas del porvenir para los futuros ingenieros mexicanos
}

\author{
F.D. Soria-Villegas y S. Hernández-García \\ Profesores de la Facultad de Ingeniería, UNAM \\ Email:francisco@dctrl.fi-b.unam.mxysilvina-hg@yahoo.com
}

(recibido: mayo de 2000; aceptado: septiembre de 2000)

\begin{abstract}
Resumen
En este trabajo se presenta una forma prospectiva de plantear la ingeniería hacia el año 2010, en donde el enfoque inicial es la manufactura. En un mundo globalizado, cambiante y de acelerado desarrollo tecnológico, se presentan 3 escenarios: el ideal, el secuencial y el pesimista; en cada uno se tratan 4 aspectos: formación curricular, vinculación escuela- industria, tecnología y empleo. El escenario ideal al año 2010 es el que permitirá tomar un camino de liderazgo en ingeniería.
\end{abstract}

Descriptores: escenario, prospectiva, certificación, acreditación, tecnología y vinculación escuela-industria.

\section{Abstract}

This article deals with a prospective approach to develop engineering activities by the year 2010, with special attention paid to manufacturing. In a global and changing world, with an accelerated technological development, it is important to put forward three scenarios: the ideal, the sequential and the pessimistic, based on four criteria, namely, education, university-industry relationships, technology, and job opportunities. The ideal scenario would result in a leadership role for engineering by the year 2010.

Keywords: scenario, prospects, certification, accreditation, technology, university-industry relationships.

\section{Introducción}

Para los escenarios se tienen variables simultáneas, aleatorias y con comportamientos no lineales que afectan a las regiones, poblaciones, estudiantes, así como a las necesidades del desarrollo creciente y el ejercicio profesional de la ingeniería en forma local y general. Como parte de las consideraciones para realizar formulaciones sobre el porvenir, se incluyen los siguientes aspectos:

a) Las tendencias en grandes regiones (la tríada); como en el continente Europeo, con la Unión Europea (UE); en el continente Asiático, con la Asociación de Naciones del Sureste Asiático (ASEAN) y en el Americano, con el Tratado de Libre Comercio (TLC); todo ello, para formar grandes bloques de desarrollo económico que seguirán modificando los factores de transformación cultural y científica, así como el uso generalizado de mercancías visibles e invisibles en las clases sociales. Dentro de la tríada, el crecimiento y desarrollo tecnológico más positivo es hacia los países de la UE; ya que para México, el TLC es un tratado comercial y financiero, en el cual se debería incluir también la colaboración científica y tecnológica.

b) Los avances a escala nacional y por regiones, en materia de acreditación de carreras, certificación de estudios, calidad educativa, ejercicio profesional, preparación continua e imagen de las instituciones públicas en beneficio de la sociedad.

c) Consideración de los planes institucionales, como el Plan Nacional de Desarrollo, a ser incluidos en cada secretaría de estado.

En cuanto a lo académico, es necesario el camino de la sustentabilidad mediante docencia e investigación de primer nivel, así como recursos adecuados para realizar futuros factibles trazados en mapas tecnológicos. Por otro 
lado, es importante marcar los requerimientos del crecimiento de la planta productiva nacional, principalmente en campos fértiles para la producción, que propicien empleos bien remunerados. Un tercer camino, son las polí. ticas y realidades sobre la planificación de la ingeniería nacional en medios como la generación de energía de la Comisión Federal de Electricidad (CFE) así como las plantas de Petroquímica Secundaria en Petróleos Mexicanos (PEMEX), para satisfacer necesidades internas y de exportación.

d) Planificar la división del trabajo y las necesidades por cubrir en el desarrollo regional, para contribuir a la formación de ingenieros y técnicos que cuenten con un alto nivel, con preparación media, o técnicos especializados en las diferentes zonas geográficas de México, según las necesidades del entorno industrial de la región; aspecto que sucede con el caso de las universidades tecnológicas para la preparación operativa y aplicaciones actuales. Por otra parte, la misión y la visión a largo plazo del capital intelectual de ingeniería de las grandes instituciones de educación como la Universidad Nacional Autónoma de México (UNAM), con preparación sólida para la innovación, el desarrollo y la investigación aplicada en ingeniería.

e) Darle mayor atención a los posgraduados como un factor que ha contribuido en los países desarrollados para el aumento del valor agregado de conocimientos en las mercancías; ya que en México, un bajo porcentaje de los posgraduados trabajan en la industria, la mayor parte labora en centros educativos, institutos y gobierno. Con 99 millones de mexicanos, se tiene que los doctores en ingeniería no llegan a 5,000 graduados en México ${ }^{1}$, de tal manera que existen pocos, comparados con las necesidades de más posgraduados en las instituciones dedicadas a las licenciaturas de ingeniería.

f) El cambio de conciencia, concepciones, uso generalizado de mercaderías y la mezcla de culturas por la información y el conocimiento, son formas de una nueva realidad en todas partes del mundo. La comercialización acentúa la producción y el uso generalizado de mercancías (globalización) en petroquímicos (las siete hermanas, Esso, Shell...), armadoras (Toyota...), equipo eléctrico (Siemens...), farmacéuticas (Bayer...), computación (HP...), comunicaciones (Motorola...), televisores

\footnotetext{
De 1971 a 1999, se tenían 366000 ingenieros titulados, según datos de la Dirección General de Profesiones. Del total de ingenieros titulados, se estima 3\% con maestría y doctorado en ingeniería en el país. En México, se gradúan, en promedio, 11 doctores en ingeniería por año.
}

(Sony...) y otros productos que se producen y consumen en distintos países del mundo.

g) Aprovechar la tecnología avanzada, la información y las telecomunicaciones que están cambiando los patrones de cultura y tradiciones milenarias. Así también, tomar en cuenta los impactos en el concepto de sociedad, de la información y el conocimiento en aquellos que tienen acceso a las diferentes tecnologías. Actualmente, es más usual para las nuevas generaciones de ingenieros, la comunicación por computadora, satélites, internet, teleconferencias y automatización a distancia.

h) Considerar que en México las exportaciones de manufactura con maquiladoras de exportación representan $90 \%$ de las exportaciones totales y $60 \%$ del movimiento económico nacional al año. El estado financiero entre los insumos importados para los productos de exportación manufacturera sin maquiladoras, son positivos y superiores a las ventas de petróleo crudo. La tecnología y la maquinaria que ocupan las grandes empresas exportadoras, en general, son importadas. Desde 1965, se tienen oportunidades en la producción de insumos para las Industrias Maquiladoras de Exportación (IME) que dependen en más del $93 \%$, de los insumos que se importan temporalmente; la mano de obra y algunos servicios son prestados por los mexicanos.

i) Analizar qué grado escolar tiene en promedio la Población Económicamente Activa (PEA) en México. La Encuesta Nacional de Empleo de 1995 del Instituto Nacional de Estadística Geografía e Informática (INEGI), publicó datos de la población mexicana, constituida por $70 \%$ de ciudadanos que no tenían estudios de secundaria terminados ${ }^{2}$. Por tanto, la visión y la capacidad de competencia abierta y prospectiva productiva están en una población que requiere mejor preparación.

j) El Consejo Nacional de Ciencia y Tecnología (CONACYT) en su plan de desarrollo tecnológico 1995-2000, presentó un estudio sobre 751 empresas en México (incluye 500 de las mayores industrias). Una de cada 3 lleva a cabo actividades de investigación y desarrollo experimental, lo cual puede ayudar a ampliar las oportunidades de vinculación escuela-industria.

\footnotetext{
${ }^{2}$ En el Cuarto Informe de Gobierno del Dr. Ernesto Zedillo, se habló del total de la PEA: $19 \%$ cursaron primaria completa, $21 \%$ primaria incompleta y $30 \%$ secundaria. De 100 inscritos en primaria, solamente terminan la licenciatura 1 o 2 (Organización para la Cooperación y el Desarrollo Económico, 1997). El promedio de años de escolaridad en 53 países de 185 que componen la ONU, México ocupa el lugar 45. Información Gaceta UNAM, Juan María Alponte, septiembre 1998, p-15, basado en The Global Competitivenes, Report 1998.
} 


\section{El escenario secuencial. Año 2010}

Las diferencias entre la economía de los países industrializados, las naciones en vías de desarrollo y la competencia comercial abierta, seguirán siendo importantes y dominadas por macro-corporaciones con divisiones. La economía mexicana tendrá mejores condiciones y oportunidades con el TLC y la UE que con otras regiones como la asiática.

La economía nacional cambiará debido a los intereses marcados por los grandes capitales, las políticas de los industriales que forman la exportación, los efectos internacionales, así como los gobernantes del país.

La deuda externa y el pago de intereses continuarán hacia el exterior, disminuyendo con ello, la liquidez para las inversiones internas, como centros públicos de desarrollo de tecnologías aplicadas para mejorar la calidad de vida de los mexicanos.

El Gobierno será rector de las políticas nacionales, seguirá el neoliberalismo y sus cambios según el entorno mundial, las privatizaciones de petroquímicas, transportes, distribución de agua y energía con adelgazamiento de su personal y automatización de procesos.

La variación promedio de crecimiento económico en México oscilará anualmente en $4.5 \%$ del Producto Interno Bruto (PIB), el cual tendrá su efecto al cubrir parcialmente los empleos demandados por ingenieros.

La formación de estudiantes para ingeniería disminuirá en porcentaje paulatinamente, siendo en términos reales, creciente, pero menor que en años anteriores por el atractivo de otras profesiones, empleos y sueldos. El trabajo para los ingenieros no se generará proporcionalmente al aumento y crecimiento de las industrias manufactureras y los servicios.

Debido a la continuidad en la gran competencia de mercancías, calidad y costos de productos terminados dentro y fuera del país, quienes no sigan adoptando la tecnología apropiada, así como las herramientas adecuadas y modernas de ingeniería en las industrias mexicanas, en pocos años podrán desaparecer o repre. sentar marcas foráneas.

El Gobierno de México continuará estimulando económicamente y con facilidades a las empresas exportadoras de productos manufacturados, pocas en comparación con las decenas de miles de empresas que producen bienes manufacturados en el país, las cuales seguirán sometidas a la competencia abierta.

Para la creación de productos maquilados, se tendrá la abundante mano de obra barata y poca ingeniería mexicana. Paulatinamente la mano de obra será desplazada por los avances en automatización y robots; asimismo, el ciclo de cambio de las IME, por nuevas localizaciones geográficas con menores costos de producción y normas ambientales suaves.

\section{Formación curricular en el escenario secuencial}

En un marco internacional, como el de México en la Organización para la Cooperación y el Desarrollo Económico (OCDE), la Organización Mundial de Comercio (OMC) y el TLC, habrá modificaciones y flexibilidad en los planes y programas de estudio en ingeniería. Se podrán cursar materias en diferentes escuelas dentro y fuera del país, crear estancias en las industrias con créditos académicos y realizar prácticas experimentales en laboratorios; lo cual creará la experiencia en el ingeniero por medio de esta vinculación escuela-industria, sumando aprendizajes e ingresos extraordinarios hacia el desarrollo sustentable en centros educativos.

El perfil y la profundidad de los contenidos en los planes de estudio en centros públicos y privados, cumplirán la misión institucional y tendrán enfoques, según la sensibilidad de quienes toman las decisiones como la rectoría, los consejos institucionales, directores, académicos y los comités consultivos externos formados por industriales. Las posibilidades curriculares se dividirán en dos grandes corrientes:

1. Continuará la formación sólida en ciencias básicas, control de contaminantes, idiomas y producciones limpias. El futuro profesionista comprenderá y resolverá los problemas de ingeniería que se le presenten. Sus estudios de licenciatura le permitirán estudiar un posgrado en el país que elija. Se formarán ingenieros eficientes y flexibles, preparados para los futuros posibles de la tecnología. La computadora y sus nuevas formas de programación se integrarán a todas las carreras de ingeniería.

2. Se apegarán hacia los sistemas productivos, los cuales formarán profesionistas técnicos con poca preparación básica en ingeniería que cubran las necesidades del presente, y así, puedan medio enfrentar los cambios vertiginosos de la tecnología. En cambio, por esta situación, aumentará su experiencia práctica y operativa en el uso de manuales. Serán bien aceptados en el sector terciario.

La certificación de estudios continuará en ascenso con los alumnos que terminen sus estudios de ingeniería y se presenten en forma opcional para obtener el testimonio de alto rendimiento académico, el cual se aplicará a las principales carreras de ingeniería; pero no todos los alumnos tendrán disponibilidad de presentar el examen global de conocimientos. 
DOI: http://dx.doi.org/10.22201/fi.25940732e.2000.01n5.024

La acreditación de carreras de ingeniería aumentará en forma secuencial. Las grandes instituciones como la UNAM, cumplirán con tener acreditadas las carreras de ingeniería. Para aumentar las acreditaciones será necesario motivàr y contratar a posgraduados. Un punto importante será el porcentaje de profesores de tiempo completo con doctorado en las carreras de ingeniería, así como tener laboratorios y cumplir con los requisitos nacionales de acreditación en un marco regional e internacional.

\section{Vinculación escuela-industria en el escenario secuencial}

La vinculación tendrá un crecimiento relativo en cuanto a proyectos y capacitación. Continuará la escasa confiabilidad de los industriales hacia posibles soluciones docentes en cuanto a sus demandas y faltarán equipos de trabajo especializados en el medio académico para resolver problemas específicos. Las inversiones económicas y la formación de posgraduados en ingeniería para equipos de trabajo preparados y flexibles, difícilmente se darán a mediano plazo.

Se proporcionarán formas y políticas de apoyo del gobierno como un actor de la vinculación escuela-industria, las cuales no se compartirán del todo hacia la intersección de la industria, las instituciones educativas y el Estado.

Los resultados concretos que solicitará la iniciativa privada a los centros educativos para la solución de un problema serán instantáneos, lo cual será imposible. En asesorías, proyectos y capacitación, se negociarán los tiempos para que parte de la oferta y la demanda queden satisfechas con una constante de tiempo de respuesta aceptable para las partes que intervengan en cada proyecto.

Se mejorarán las conexiones por redes de computo con otras instituciones, empresas, organismos públicos, científicos y medios que ayuden a ampliar el flujo de información para crear conocimiento, innovar teorías, ampliar aplicaciones y visiones que orienten el trabajo de los académicos, su desarrollo, investigación y procesos cuyos objetivos serán la búsqueda y materialización del conocimiento.

\section{Tecnología en el escenario secuencial}

En materia prima y partes para la producción nacional y de exportación manufacturera, las balanzas tecnológica y comercial serán negativas para México. El balance neto entre importaciones de insumos para productos de exportación y las ventas de manufactura al extranjero serán favorables al país. Las importaciones de la manufactura para consumo nacional aumentarán.

Efectos sobre la Investigación y Desarrollo (I \& D) serán la formación de pocos centros de desarrollo e investigación para resolver necesidades de la iniciativa privada, de igual manera, sucederá con los centros educativos privados.

En el caso de los institutos y los centros de investigación y desarrollo públicos se seguirán dos caminos: la incorporación paulatina a la iniciativa privada y lo sustentable; ya que al obtener ingresos por proyectos externos, los investigadores tendrán la oportunidad de resolver problemas de las industrias teniendo ingresos adicionales a su salario base. La investigación básica disminuirá para dar paso a la aplicada.

En algunas instituciones educativas se iniciará el desarrollo de laboratorios experimentales.

En desarrollo tecnológico, las mejores oportunidades se presentarán para los ingenieros con un alto rendimiento académico, incluyendo idiomas y experiencia. Sin embargo, la automatización y los diseños tecnológicos para los procesos de las grandes compañías se seguirán realizando en las empresas matrices.

Para el año 2010 aumentarán los puestos de trabajo para los técnicos, todos serán más dependientes de la tecnología extranjera, disminuirán las oportunidades para ingenieros con preparación media, la cantidad de profesionistas egresados crecerá más que la economía nacional.

La parte de la población de ingenieros y posgraduados que estarán dedicados a la creación de nuevos bienes y servicios será deficiente; habrá empleo solamente para ventas, representaciones de empresas extranjeras, y en general, servicios. En algunos casos, para contrataciones, se les pedirá el posgrado.

\section{Empleo en el escenario secuencial}

Con el crecimiento promedio del PIB se crearán alrededor de 700000 empleos anuales de todo tipo, de los cuales menos de $2 \%$ será para los ingenieros, propiciando así mayor demanda que apertura de plazas para trabajar en las industrias.

El ingeniero que por alguna razón pierda su empleo para el año 2010, difícilmente encontrará otro trabajo con buen salario y expectativas de ir escalando mejores niveles de puesto; algunos se dedicarán a los servicios de otro tipo. Para cada empleo de ingeniería habrá muchos aspirantes y para lograr su ocupación en grandes empresas será nece. sario contar con el título y cédula profesionales, un idioma diferente al materno, la constancia de testimonio de alto rendimiento académico expedido por un organismo como el Centro Nacional de Evaluación de la Educación Superior (CENEVAL) y ser aceptado en las entrevistas.

Los ingresos de quienes apenas terminaron la carrera, continuarán siendo suficientes para lo indispensable, poco dinero para un nivel de vida con alta calidad y para un 
profesionista que estudió 17 años para obtener su título como ingeniero.

Las oportunidades laborales se otorgarán más en las PYMEs, éstas tendrán menores recursos que las grandes empresas para pagar sueldos elevados y asegurar su ascenso a niveles ejecutivos y de carácter internacional. Las grandes empresas seguirán automatizando e incorporando más robots.

Para los empleos de técnicos en las IME y las manufacturas, se tomarán en cuenta los campos de especialización productiva y la flexibilidad de adaptación tanto a la producción masiva, como a los productos diferenciados. La mano de obra abundante de ingenieros, disminuirá en función de los avances en automatización.

En el marco del TLC y con acuerdos entre estados, la movilidad profesional será convenida. Para que los ingenieros mexicanos trabajen en localidades de EUA o Canadá deberán cumplir con el título expedido por una institución con carrera acreditada nacionalmente; hablar, escribir y entender un idioma extranjero, haber tenido un promedio mínimo de 9.0 y estar certificado en México.

Los empleos seguirán dependiendo de factores generales como el desarrollo regional, la escolaridad, el movimiento de capitales en la economía nacional; las inversiones directas con visión y planificación, el aumento de industrias en bienes y servicios, la inflación, el interés por prestamos y el PIB.

\section{El escenario pesimista. Año 2010}

La economía nacional será semi-estable debido a la dependencia de las inversiones extranjeras, el aumento de la deuda externa, las compras de tecnología extranjera, las grandes importaciones y las variaciones a la baja de los precios del petróleo crudo. Se privatizará la petroquímica secundaria y la petroquímica primaria tendrá participación privada. Los grandes ingresos de impuestos que ahora recibe el Gobierno por concepto de PEMEX disminuirán notablemente. Una de las 20 economías más grandes a nivel mundial, como lo es la mexicana, seguirá afectada por los centros financieros internacionales, como pasó con el efecto brasileño y el asiático; de igual manera, el impacto perjudicará los cambios políticos de grupos poderosos y las directrices del Presidente para fines del año 2010.

En ofertas y demandas de ingenieros, habrá pocos empleos y muchos aspirantes. La remuneración profesional seguirá siendo inadecuada en la mayoría de los niveles, ya que por más de tres salarios mínimos iniciarán el trabajo los recién egresados en actividades que se confundirán con las realizadas por técnicos.

La inflación, la paridad del peso respecto al dólar y la canasta de monedas fuertes, seguirán cuesta debajo de acuerdo a nuestra moneda, la economía mexicana prácticamente estará dolarizada. Subir en la escala social y ofrecer a la familia una mejor calidad de vida será difícil de cubrir en el medio de los ingenieros.

El consumo de productos y servicios en el mercado interno, así como la producción, crecerán poco; prácticamente estarán en equilibrio, disminuirá la capacidad de compra, los costos de producción manual aumentarán y algunas compañías tendrán que cerrar, otras, esperar mejores tiempos para abrir sus puertas.

Habrá oportunidad para un grupo de mexicanos que cuenten con compañías de productos de exportación, las cuales seguirán siendo buenos negocios, así como generadoras de empleos e ingreso de divisas al país. Algunos productores nacionales también serán favorecidos, ya que la elevación de sus productos foráneos por la devaluación del peso respecto al dólar, les permitirá vender más mercancía dentro y fuera del territorio nacional.

En términos reales la economía mexicana crecerá, pero no será suficiente para las necesidades del índice de natalidad de $1.5 \%$ anual, que sumado a los miles de jóvenes que terminarán sus estudios cada año, más los rezagados por el desempleo en edad de trabajar, no tendrán oportunidades de un empleo digno. Así también, su ascenso en la escala social será difícil.

Las grandes compañías extranjeras se seguirán instalando en México, traerán sus procesos, ingenieros y maquinaria, los ingenieros mexicanos que se ocuparán serán pocos, su función será más bien para los servicios, mano de obra y mantenimiento industrial.

\section{Formación curricular en el escenario pesimista}

Es posible un país maquilador, la ingeniería será más de servicios que de nuevos productos, realidad que algunos no quisieran pensar para México. Por ello, las nuevas generaciones no requerirán de grandes bases científicas, lo cual generará una mejora temporal en el reconocimiento social.

La formación curricular continuará sin grandes cambios en los aprendizajes tradicionales, el mantener los programas significará atraso o aplazamiento para aprender las nuevas tecnologías. Habrá mejor aprendizaje en laboratorios, uso de paquetes de computadora y estancias en las industrias. La parte experimental será profunda, de tal manera que los futuros ingenieros serán menos teóricos, sus conocimientos y habilidades les permitirán flexibilidad en los empleos con perfiles más enfocados hacia el trabajo de mantenimiento, administración y ventas que de innovación y creación de nuevas mercancías; es decir, más hacia las líneas de producción establecidas con un mejor nivel que los técnicos, pero con más habilidades prácticas que los egresados de las pasadas generaciones de ingeniería. 
Se continuará asignando la cédula y título profesionales. El Centro Nacional de Evaluación de la Educación Superior tendrá pocos alumnos que aspiren y accedan a evaluar sus conocimientos y habilidades para tener el testimonio de alto rendimiento académico; solamente algunas compañías pedirán la certificación del egresado.

Las carreras de ingeniería, en general, no estarán acreditadas; pocos directivos de las instituciones sentirán la necesidad de hacerlo, algunos comentarán que existen formas irregulares para obtener la acreditación y pedirán esperar tiempos mejores; así como estabilidad en los requisitos. Los requerimientos de los empleadores serán satisfechos con los egresados, lo más importante será la técnica aplicada.

\section{Vinculación escuela-industria en el escenario pesimista}

La vinculación con las instituciones nacionales, las empresas y el Estado estará prácticamente igual que ahora, es decir, con proyectos de organismos gubernamentales (pocos por las privatizaciones) y unos cuantos de las industrias privadas. La sociedad continuará desconociendo la amplia labor docente, seguirá la desconfianza de los industriales y los presupuestos provocarán que personal docente busque la vinculación, pero con escasos resultados.

La escasa difusión del desarrollo tecnológico seguirá por el mismo camino, sus resultados poco conocidos por la sociedad le darán nuevamente cuestionamientos duros sobre la docencia, investigación, inversiones y los resultados de proyectos. Habrá falta de reconocimiento de la sociedad sobre la vinculación de instituciones educativas con las industrias.

Las patentes serán pocas en México, las normas internacionales vendrán de los países más avanzados, la vinculación industrial se dará con los centros de investigación de las grandes empresas en distintos lugares geográficos del extranjero, las soluciones tecnológicas para las PYMEs serán comprar al exterior, la balanza tecnológica tendrá números rojos.

Los análisis sobre las mejores opciones seguirán con preguntas como las siguientes ¿Qué líneas de investigación serán las más útiles para las escuelas y las industrias? ¿Cómo lograr financiamientos para las instituciones educativas? ¿Cuál sería el perfil adecuado del personal docente para la vinculación? ¿Cómo difundir la vinculación? ¿Qué hacer para concertar acciones en el ámbito nacional y extranjero? ¿Cómo involucrar a la sociedad en la vinculación escuela-industria?

Existirá la inercia para superar el estado de la vinculación escuela-industria, seguirán los mismos usos y costumbres del personal docente, industriales y gobierno. La iniciativa privada no estará interesada en realizar capacitación, ni actualización; comprará paquetes tecnológicos y proyectos "llave en mano" a los extranjeros. La economía nacional limitará el mercado interno y las oportunidades de vinculación con la academia.

Tendremos recursos humanos de nivel técnico e ingenieros prácticos con poca capacidad creativa y de invención, sin liderazgo, con poca energía para resolver problemas; con ausencia de conocimientos profundos para afrontar problemas mayores, en la perspectiva tecnológica.

En parte del personal docente, habrá sentimientos de aislamiento y desmembramiento por las oportunidades comunes en cuanto a la solución de problemas entre instituciones educativas e industriales.

\section{Tecnología en el escenario pesimista}

La dependencia tecnológica continuará en forma sostenida (los números rojos aumentarán), pero no estaremos en recesión. Las empresas manufactureras y de servicios continuarán importando materia prima, productos y bienes de capital para sus producciones.

Los equipos, componentes y personal especializado vendrán del extranjero, la ingeniería mexicana será más para selección de equipo, ventas de marcas extranjeras y de mantenimiento, dejando atrás las posibilidades del diseño de nuevos materiales, bienes de capital, manufacturas de consumo general, creadas, diseñadas y producidas dentro del país por mexicanos.

Se seguirán consumiendo mercancías con respaldo internacional, como equipos de telecomunicaciones y computadoras, que se armarán y venderán dentro y fuera de las fronteras nacionales; dando la apariencia de ser generadores de tecnologías.

Las empresas exportadoras seguirán siendo líderes en el país, sus formas de producción de calidad con normas internacionales serán aceptadas en el extranjero. Los beneficios seguirán dando ingresos de divisas al país y creación de empleos. La exportación recibirá apoyo de los gobernantes y los consumidores extranjeros estarán satisfechos de los productos mexicanos.

En México disminuirán los centros de investigación y desarrollo que ahora forman parte de las instituciones públicas. La iniciativa privada de las grandes y medianas empresas realizará sus proyectos por medio de sus compañías, pocos organismos nacionales públicos realizarán I\&D aplicados.

\section{Empleo en el escenario pesimista}

La globalización continuará, los costos de vida estarán homologados respecto a otros países, las clases privilegiadas 
podrán comer bien tres veces al día y tener una calidad de vida aceptable. El bienestar se concentrará en menos de $15 \%$ de los mexicanos. El ingreso por trabajo y la economía de los ingenieros los llevará a situaciones de preservar el empleo sobre la familia.

La economía nacional crecerá anualmente 3\% del PIB en promedio, superior al índice de natalidad, lo cual dará oportunidad a la creación de empleos insuficientes. Los perfiles de los puestos serán hacia la parte administrativa, operativa, el mantenimiento y los servicios, habrá intersección entre quienes sean técnicos y los ingenieros, los pocos puestos podrán ser ocupados en forma indistinta. Habrá más egresados que empleos requeridos, algunos ingenieros encontrarán otras altetnativas, hasta ocupaciones en la economía subterránea con el consecuente deterioro de los conocimientos adquiridos en su formación educativa.

El encontrar trabajo y hacer carrera, será difícil. Quienes tengan trabajo serán afortunados a pesar de ganar poco, quienes no lo tengan o los despidan tendrán problemas para encontrar otro. La inseguridad aumentará.

Los productos maquilados de exportación continuarán creciendo a un índice mayor al PIB promedio del país, por tanto, disminuirá la brecha entre la cantidad de los empleados del sector manufacturero y los que laboran en las maquiladoras de exportación. La mayor cantidad de puestos de trabajo en las IME serán de mano de obra, pocos ingenieros con ocupaciones en mantenimiento y la administración productiva.

Las uniones y las fusiones continuarán en la industria, habrá mayor cantidad de mercancías que entren al país, de tal manera que los empleos decrecerán como efecto de la disminución de las producciones internas, ya que al tener bienes y servicios extranjeros de menor costo que los productos nacionales, será más fácil que subsistan los que logren abatir costos en bienes a escala internacional.

\section{El escenario ideal. Año 2010}

México formará parte de las 10 economías más fuertes del mundo desarrollado. La moneda mexicana será fuerte, la inflación será de $0.5 \%$ anual; el peso formará parte de la canasta de monedas internacionales.

Se tendrán políticas adecuadas al desarrollo nacional, la economía estará industrializada con nuevas aplicaciones en petroquímica, biocombustibles, electroquímica, nuevas herramientas moleculares para descifrar y reproducir el genoma, uso de la glucosa para polímeros biodegradables, biocelulosa, petroquímicos, en superconductores, biotecnología, nanotecnología electrónica, microsatélites, optoelectrónica avanzada, computación evolutiva, fluidos complejos, redes neuronales, tecnología de comunicaciones, realidad virtual, programación avanzada y otros aspectos. Los medios de producción que ahora tiene el gobierno estarán privatizados y desarrollarán tecnología propia en comunión con la vinculación escuela-industria.

La deuda externa e interna, disminuirán por la sana y buena administración pública, aumentarán los financiamientos debido a inversiones privadas nacionales con buenos resultados, la inversión extranjera directa aumentará notablemente, los bancos prestarán a bajos intereses, grandes industrias y centros de desarrollo tecnológico se instalarán en el país. El ingreso petrolero ayudará a impulsar exponencialmente a la infraestructura del país; tendremos políticas y realidades compatibles con el bienestar social.

El desarrollo de la economía nacional traerá la necesidad de más ingenieros, quienes no serán cubiertos por egresados nacionales. Todos los ingenieros adquirirán trabajos estables con buenos sueldos; los maestros y doctores laborarán en centros para desarrollos aplicados a producciones de mercancías y nuevos materiales; los bienes nacionales serán flexibles y con mayor valor agregado de conocimientos, el empleo crecerá más que la población en edad de trabajar, nadie se quedará sin puesto de trabajo, todos los mexicanos estaremos satisfechos de las industrias, del gobierno y de la calidad de vida.

Aumentarán notablemente los movimientos económicos de las exportaciones, en particular, las vías marítimas, los puertos se modernizarán, los volúmenes de cargas que entrarán y saldrán del país serán sorprendentes. Producciones como los derivados del petróleo, realizado y producido por mexicanos, se exportará a países europeos, naciones asiáticas de la cuenca del Pacífico y otras ciudades en América Latina.

\section{Formación curricular en el escenario ideal}

Todos los estudiantes de licenciatura tendrán una beca con un salario mínimo (apoyo de la industria mexicana) y no pagarán colegiatura, debiendo cumplir condiciones de excelencia en conocimientos y calificaciones. Todos tendrán trabajo al terminar sus estudios.

Los currículos de ingeniería serán flexibles; todos los estudiantes tendrán un tutor que podrá autorizar que cursen materias en otras facultades y universidades dentro y fuera del país, habrá créditos por experiencias profesionales (sector público y privado) y se podrán cursar materias de otras carreras. Cumpliendo los créditos y requisitos del currículum, se tendrá el título y la cédula profesionales, y no habrá duda en la calidad educativa.

Desde el año 2005 será requisito indispensable para iniciar las carreras de ingeniería, que los estudiantes presenten exámenes de comprensión en dos idiomas. 
Hacia el año 2010 las evaluaciones de idioma serán efectuadas además de comprensión, en escuchar, interpretar, analizar y hablar de preferencia dos idiomas.

Las ciencias básicas se reforzarán y se sumarán en forma obligatoria a materias relacionadas con las tecnologías para producciones limpias, el cuidado del medio ambiente y la necesidad de racionalizar los recursos naturales para beneficio de la humanidad. Algunas carreras como computación se transformarán para fusionar campos como la información y las telecomunicaciones. La computación estará incluida en todas las carreras de ingeniería.

Los laboratorios con plantas piloto en las escuelas y facultades, tendrán los equipos básicos para el desarrollo de prácticas y experimentos, así como la formulación de teorías que sean orientadas por los profesores y les permitan la comprobación y los errores en las consideraciones. El pensamiento creativo y los mapas mentales serán formas para llevar a efecto los cursos en laboratorios, según la capacidad de cada estudiante.

Los estudiantes aprenderán a aprender por sí mismos en forma permanente. El análisis, síntesis, la creatividad y las patentes obligatorias por cada egresado serán parte fundamental de los profesionistas del año 2010.

Las fronteras tecnológicas no existirán, el libre transito de los profesionistas por medio de certificados internacionales será una realidad. Estudiar en instituciones que tengan la acreditación de carreras será también una carta de presentación, los científicos mexicanos tendrán la misma calidad de los que trabajen en países desarrollados.

La mayor parte de egresados de ingeniería continuarán sus estudios de posgrado hacia doctorado, de tal manera que se tendrán edificios de investigadores de primer nivel; habrá aldeas de científicos que se financiarán en campos seleccionados por el sector privado, el cual incorporará a sus procesos nuevos avances tecnológicos.

\section{Vinculación escuela-industria en el escenario ideal}

Con la vinculación, los escenarios sobre desarrollo tecnológico mejorarán en las regiones del territorio nacional, se acortará el tiempo para generar centros de desarrollo experimental y se tendrán nodos y redes con científicos que colaboren en proyectos escuela-industria. Con cambios tecnológicos rápidos, se redefinirán las fronteras mentales, se tendrán nuevas y diferentes mercancías, se multiplicarán las opciones creativas, productivas y de comercialización abierta, en cuyo contexto se reflexionará objetivamente, así también se tomarán decisiones que competirán en los mercados mundiales.

Se formará una cultura científica acerca de la vinculación escuela-industria y será fundamental para los empresarios y el gobierno, la intersección de oportunidades de desarrollo con centros educativos y de I \& D.
La radio y la televisión difundirán gratuitamente la vinculación. Aprenderemos de los científicos mexicanos y de los extranjeros, se formarán masas críticas de ingenieros que semejantes a las esponjas absorberán los conocimientos y las experiencias sobre nuevas formas de desarrollo tecnológico.

Con el estandarte de la vinculación, crecerán las nuevas generaciones de ingenieros forjados como el acero, con la fuerza del liderazgo, del cambio continuo hacia nuevos materiales, elaboración de prototipos, nuevas máquinas, nuevos esquemas para planear los servicios y los bienes materiales producidos en México.

Los campos de trabajo serán flexibles, se identificarán necesidades y se desarrollarán bienes y servicios en los centros educativos. Todo el personal académico tendrá buenos salarios y becas adicionales. Las instituciones educativas tendrán centros regionales de innovación tecnológica, aldeas de científicos mexicanos, todos trabajarán en equipo; se tendrán redes que ofrecerán y manejarán las ofertas y demandas de los industriales y de quienes están trabajando en los proyectos.

Todas las empresas ofrecerán estancias a estudiantes y académicos con las mejores condiciones. Los industriales seleccionarán a los mejores alumnos para formar centros de emprendedores. Instituciones y empresarios generarán equipos de especialistas preparados para los futuros alternativos que generen escenarios deseables, planifiquen, y orienten adecuadamente a las empresas hacia resultados óptimos para un futuro deseado.

\section{Tecnología en el escenario ideal}

Los centros docentes, de investigación privados y públicos tendrán ingresos suficientes por el pago de proyectos externos, la mayor parte de gastos de su sostenimiento serán por la realización de trabajos por vinculación escuela-industria. La sociedad reconocerá ampliamente al personal docente e investigadores por su trabajo.

Los centros públicos de I \& D sostenidos exclusivamente del presupuesto del Gobierno de México serán pocos, la mayor parte serán sustentables, teniendo más ingresos que egresos, dando oportunidad a que la iniciativa privada los compre y abra nuevas zonas para formar centros de desarrollo financiados exclusivamente para la creación de nuevas mercaderías. Habrá personal especializado en los centros de investigación y docencia con la misión de construir el puente con las industrias, en particular, hacia las PYMEs y las grandes industrias.

Las industrias privadas en conglomerados, formarán centros de investigacion y desarrollo para resolver sus problemas en comunidad. Tendrán éxito las empresas integradoras, que sumadas a las grandes empresas tendrán 
aldeas de científicos para soluciones con tecnologías apropiadas a procesos y productos.

Las grandes inversiones propiciarán que en compañías de computación, instrumentos inteligentes, artículos electrónicos, electrodomésticos y otras, requieran durante algún tiempo la mano de obra; sin embargo, iniciarán la formación de centros de I \& D para automatizar líneas de producción flexible y continuar hacia nuevas mercancías para los mercados internos y de exportación.

Las vías de exportación e importación se ampliarán para propiciar polos de desarrollo en todo el país. La ingeniería para su construcción y operación se realizará en México.

La fabricación de más y nuevas mercancías con certificados de origen para exportaciones y demanda interna, propiciarán el aumento de la planta productiva. Un 5\% de las ganancias de los industriales se invertirá en I \& D de nuevos bienes. Se generarán empleos de todo tipo, iguales a los de países con gran desarrollo industrial.

La tecnología mexicana dará paso a la primer generación de autómatas programables, "tontos" pero activos, esos mecanismos aprenderán y serán una realidad que hará disminuir el trabajo pesado y rutinario.

\section{Empleo en el escenario ideal}

El desempleo se abatirá hasta valores negativos, todos los mexicanos en edad de trabajar tendrán posibilidades de empleo permanente; para los puestos vacantes, se solicitará el apoyo de extranjeros. Todos recibirán becas para estudiar y el nivel académico de los mexicanos será mayor a 15 años de educación escolar.

Se formarán centros de producción con ingenieros especializados en producciones regionales y rurales. Las personas que emigraban del campo a las ciudades nuevamente regresarán para la producción del sector primario y mejoramiento en su calidad de vida.

El crecimiento del país por medio de las inversiones con ingenieros de primer nivel, ofrecerá las oportunidades para adquirir y producir bienes de capital con patentes y fabricación nacional. Se contará con mejores oportunidades de adquirir bienes de capital nacional. Las industrias producirán a menor costo, habrá más mercancías diferenciadas, de alta escala y calidad internacional; se requerirán miles de ingenieros anualmente, los cuales realizarán todos los niveles de funciones en ingeniería, desde la línea de producción hasta los puestos ejecutivos.

Las actividades laborales del sector primario serán compatibles con los estudios de ingeniería, con aplicaciones de conocimientos, diseños y toma de decisiones sobre aspectos ligados a los sistemas productivos; se tendrá una base de técnicos para resolver problemas de nivel medio en las empresas.
La producción se extenderá hasta los medios de transporte marítimo, los ingenieros desarrollarán todo tipo de sistemas automatizados para cargar, descargar y optimizar los puertos, por tanto, los tiempos de entrega de mercancías disminuirán, los costos bajarán y los ingresos aumentarán.

Los centros educativos, y de $I \& D$ de la iniciativa privada y pública, cumplirán con la investigación teórica y aplicada como un país desarrollado, contribuyendo a extender los límites del conocimiento. Se tendrán cuerpos de académicos e investigadores dedicados a campos de desarrollo experimental para resolver problemas de los sectores público y privado. Industriales extranjeros comprarán patentes a mexicanos y solicitarán que se resuelvan problemas tecnológicos de primer nivel.

El desarrollo del trabajo de los ingenieros podrá realizarse lejos de los centros urbanos, en un medio ambiente totalmente limpio.

\section{Conclusiones}

El trabajo prospectivo de la ingeniería y sus campos tratados es una forma de presentar alternativas sobre el porvenir. Estamos seguros de que los escenarios deben ser de una manera que permita realizar la planeación a largo plazo, cumpliendo metas parciales y orientando el camino hacia el futuro deseable de la ingeniería en México en competencias abiertas.

Los temas expuestos tienen variables simultáneas, que son dependientes, dinámicas, no lineales, que están correlacionadas y se deben estudiar y probar detenidamente en el transcurso del tiempo. Todo ello, para esperar condiciones estables que permitan disminuir la incertidumbre y realizar de una manera cuantitativa la planeación con las condiciones que aseguren llegar a un futuro deseado. Esto se llevará a cabo como sucede con un semáforo: verde para continuar el camino, ámbar para orientar nuevamente el plan y rojo para corregir la planeación y quizás la meta.

En preparación académica, se necesitará la certificación de alto rendimiento académico en los egresados de licenciatura, más carreras que cumplan con la acreditación y la continuidad permanente del aprendizaje; desarrollar la economía de la industrialización para abrir oportunidades de trabajo a todos los profesionales de la ingeniería, preparando y aprovechando a mucho más posgraduados, quienes se encarguen de cambiar la cultura de trabajo y los resultados en la vinculación escuela-industria.

El desarrollo de la tecnología e investigaciones aplicadas será esencial para el crecimiento y aumento de las exportaciones, así como de las industrias nacionales, con sus consecuencias positivas en la generación de empleos en ingeniería y el mejoramiento del bienestar de la sociedad en que vivimos. 
La independencia tecnológica en campos específicos sólo se podrá lograr con políticas nacionales de largo plazo, aumentando las zonas industriales en las diferentes regiones del país, con medios y personal altamente calificado; sumado a la cultura de la creatividad y la materialización del conocimiento a cada ingeniero, que trabajando en equipo, podrá sumarse al desarrollo tecnológico para beneficio de la sociedad.

Existen y existirán muchas interrogantes sobre los temas expuestos y el porvenir deseado para los mexicanos. Las puertas están abiertas para debatir y concluir sobre las posibilidades de mejoramiento en la preparación de los ingenieros, acerca de cuántos se dedicarán al desarrollo de la tecnología y también sobre la seguridad de empleos permanentes con buenos sueldos y con el compromiso del sentido social de contribución hacia la modernización productiva nacional dentro de un ambiente de globalización y tendencias dirigidas por los grandes líderes mundiales.

\section{Bibliografía recomendada}

Ackoff-Russel (1999). Rediseñando el futuro. Limusa, México. Aguirre-Vázquez, et.al. (1997). El futuro de la vinculación empresa-educación superior una experiencia mutua de aprendizaje. IIMAS, UNAM, México.

Arguelles A. (1998). La educación tecnológica en el mundo. Limusa, México.

Álvarez J., et al. (1995). Aspectos tecnológicos de la modernización industrial de México. Editorial FCE, México.

ANUIES (2000). La educación superior en el siglo XXl. ANUIES, México.

Campos M.A., et al. (1994). Universidad y vinculación: nuevos retos viejos y problemas. IIMAS, UNAM, México.
Cervera M. (1996). Globalización japonesa, lecciones para América Latina. Editorial Siglo XXI, México.

Chavero-González A., et al. (1996). Vinculación Universidad, Estado, producción; El caso de los posgrados en México. Editorial Siglo XXl, México.

Edvinson L. y Malone M. (1998). El capital intelectual. Grupo Editorial Noria.

Facultad de Ciencias Políticas y Sociales, UNAM (febrero-abril, 1999). Los escenarios en el umbral del tercer milenio; análisis prospectivo. Seminario del Doctorado, México.

Fondo de Intercambio Académico AC. (1997). Educación, productividad y empleo. México.

IIEC, UNAM (5 y 6 de septiembre, 2000). El futuro de la ciencia y la tecnología. Seminario de economía , ciencia y tecnología, México.

Kennedy P. (1993). Hacia el siglo XXl. Plaza \& James, Barcelona, España.

Lee A.E. (1998). Engineering and Education for the Future. IEEE Spectrum, enero, 77-85.

Mulas P. (1995). Aspectos tecnológicos de la modernización industrial de México. Fondo de Cultura Económica, México.

OCDE (1997). Exámenes de las políticas nacionales de educación en México. México.

Santa María-Ambrís R. (1995). Los desafíos del posgrado en América Latina. Colección UDUAL, México.

Solana F. (1999), Educación en el siglo XXl. Noriega Editores, México.

Universidad Autónoma Metropolitana (UAM,Verano 98), Escenarios de la globalización, UAM Xochimilco. Política y Cultura, No. 10 .

Unión Panamericana de Ingeniería (2000). El ejercicio profesional de la ingeniería y el Siglo XXl. XXVl Convención de UPADI, Panamá, agosto.

\section{Semblanza de los autores}

Francisco Daniel Soria-Villegas. Titulado en la Facultad de Ingeniería, UNAM como ingeniero mecánico electricista en 1976 y como maestro en ingeniería en 1995, cursó un diplomado en comercio exterior en 1997, en la División de Educación Continua de la Facultad de Ciencias Políticas y Sociales, UNAM. Ha publicado y presentado ponencias sobre prospectiva de la ingeniería eléctrica hacia el año 2010, así como de los campos de instrumentación y control de procesos. De 1997 a 1999, formó parte del comité de ingeniería del centro nacional de evaluación de la educación superior. Actualmente integra el comité técnico para la enseñanza de la ingeniería en México y es profesor titular de tiempo completo en la Facultad de ingeniería de la UNAM.

Silvina Hernández-García. Titulada como ingeniera mecánica electricista en 1981; obtuvo el grado de maestra en ingeniería en 1999, en la Facultad de Ingeniería de la UNAM. Es consultora de proyectos de innovación tecnológica del Consejo Nacional de Ciencia y Tecnología. Ha desarrollado diferentes proyectos de vinculación escuela-industria y cuenta con varias publicaciones sobre educación, ingeniería industrial e ingeniería de producción. Actualmente es coordinadora del diplomado de Ingeniería de Producción en la División de Educación Continua de la Facultad de Ingeniería, UNAM. 